\title{
Confidence and Construal Framing: When Confidence Increases versus Decreases Information Processing
}

\author{
ECHO WEN WAN \\ DEREK D. RUCKER
}

\begin{abstract}
A large literature demonstrates that people process information more carefully in states of low compared to high confidence. This article presents an alternative hypothesis that either high or low confidence can increase or decrease information processing on the basis of how information is construed. Five experiments demonstrate two sets of findings supporting this alternative formulation. First, low confidence leads people to focus on concrete construals, whereas high confidence leads people to focus on abstract construals. Second, people in a state of low confidence view messages framed in a concrete manner as more relevant and thus engage in greater processing of messages framed concretely; in contrast, people in a state of high confidence view messages framed in an abstract manner as more relevant and thus engage in greater processing of messages framed abstractly. These results enrich the literature by providing a fundamental shift in understanding how psychological confidence influences information processing.
\end{abstract}

$\mathrm{G}$ arnering consumers' attention to information is often a key goal of advertising and marketing efforts (Pieters and Wedel 2004; Rucker and Petty 2006). Indeed, a large literature testifies to the fact that increased attention to communications with compelling arguments enhances persuasion (e.g., Petty and Cacioppo 1986; Petty and Wegener 1998; Rucker, Petty, and Priester 2007). As such, an important goal of past research has been to understand variables that increase or decrease information processing. For example, consumers attend more to information when the information is personally relevant, when there are incentives

Echo Wen Wan (ewan@business.hku.hk) is associate professor in marketing, School of Business, the University of Hong Kong, Pokfulam Road, Hong Kong. Derek D. Rucker (d-rucker@kellogg.northwestern.edu) is associate professor at the Kellogg School of Management, Northwestern University, 2001 Sheridan Road, Evanston, IL 60208. The authors gratefully acknowledge the insightful comments from the editor, the associate editor, and the three reviewers. They also thank Brian Sternthal for his helpful comments. This research was supported by a Hong Kong Special Administrative Region Research Grants Council research grant (HKU 755511) awarded to the first author and the Richard Clewett Research Professorship awarded to the second author.

Ann McGill served as editor and Jaideep Sengupta served as associate editor for this article.

Electronically published June 22, 2012 to do so, or when they naturally enjoy thinking (for a review, see Petty and Wegener 1998).

The general degree of confidence that people experience on the basis of either chronic or situational factors is known to govern consumers' information processing (Kruglanski 1989; Petty et al. 2007). High confidence is a state of feeling sure and certain about one's environment, actions, and ideas, whereas low confidence, or doubt, is a state of feeling unsure and uncertain about one's environment, actions, and ideas (Kruglanski 1989; Tormala, Rucker, and Seger 2008). A number of papers find that chronic or situationally induced states of low confidence lead to more information processing than do states of high confidence. For example, Weary and Jacobson (1997) found that people chronically uncertain of the causes of outcomes in their environment engaged in greater information processing than those chronically confident of causes of outcomes. Similarly, Briñol et al. (2007) found that relative to individuals in a state of high power, which was associated with high confidence, individuals in a state of low power, which was associated with low confidence, were more influenced by whether the merits of an argument were strong (i.e., compelling) or weak (i.e., specious), a classic measure of processing (see Petty and Cacioppo 1986; Petty and Wegener 1998; Rucker et al. 2007). Tiedens and Linton (2001) also found that people engaged in greater processing when induced with emotions associ- 
ated with low confidence (e.g., worry) than when induced with emotions associated with high confidence (e.g., anger).

The main explanation for these past findings is that even incidental states of low confidence prompt individuals to infer that they have insufficient knowledge in a given situation, which motivates them to compensate by systematically seeking out and processing information (Chaiken, Liberman, and Eagly 1989; Maheswaran and Chaiken 1991; Wood and Lynch 2002). In contrast, high confidence leads people to infer that their knowledge is sufficient and there is no need to carefully process additional information, which prompts heuristic processing (Maheswaran and Chaiken 1991). In the current research, we question how pervasive and unwavering the association between low confidence (high confidence) and increased (decreased) information processing is. We propose that a crucial moderator between consumers' level of confidence and information processing is the level of abstraction at which a message is framed.

\section{REVERSING THE INFLUENCE OF CONFIDENCE ON INFORMATION PROCESSING}

Although the majority of past research is consistent with the proposition that states of high confidence decrease information processing, there is a minority report suggesting that this association is not set in stone. For example, Correll, Spencer, and Zanna (2004) showed that self-affirmation enhanced confidence and led to a greater sensitivity to weak versus strong arguments, presumably because self-affirmation enhances one's objectivity. Albarracín and Mitchell (2004) found that people highly confident in their ability to defend their attitudes processed counterattitudinal information more carefully than those with low defensive confidence because high defensive confidence led people to believe they could defend their attitudes against counterarguments successfully. These findings form an initial crack in the long-established foundation between confidence and information processing (see also Tormala et al. 2008).

In the current work, we propose that the psychological states of high and low confidence might evoke qualitatively different types of thinking with respect to their level of construal (Trope and Liberman 2003). In particular, we put forth three specific hypotheses. First, states of high confidence tend to lead consumers to think at a more abstract level of construal, whereas states of low confidence tend to lead consumers to think at a more concrete level of construal (Trope and Liberman 2003). Second, differences in construal naturally orient people to focus on, and view as relevant, different types of information. Low confidence will lead people to view a message framed in a concrete manner as more relevant, whereas high confidence will lead people to view a message framed in an abstract manner as more relevant. Third, as a result of this difference in relevance, message processing will be affected by the relation between consumers' confidence level and the message frame. When a message is framed in a concrete manner, the "classic" effect of confidence will emerge (i.e., more processing under low confidence than high confidence). In contrast, when a message is framed in an abstract manner, the effects of confidence will reverse (i.e., more processing under high confidence than low confidence).

In testing these hypotheses, the current article makes two broad contributions to the literature. First, a novel relationship between psychological confidence and construal level is documented. Second, confidence is shown to produce both less and more information processing, depending on whether information is framed in a concrete or abstract fashion.

\section{THEORETICAL BACKGROUND}

\section{Psychological Confidence and Construal Level}

According to research on psychological confidence (Chaiken et al. 1989; Wood and Lynch 2002), being confident means consumers feel that they have the necessary knowledge and skills for handling an issue, or they know that a course of action is correct, and outcomes can be accurately predicted. This feeling of certainty signals a benign situation in which people have a low need to engage in piecemeal understanding of information. Consistent with this notion, past research on information processing has suggested that perceiving a situation as benign often triggers top-down processing (Schwarz 2002, 2004; Sujan 1985), and topdown processing, in turn, has been suggested to be guided by high-level, abstract concepts and schematic information (Johnson 1984; Park and Smith 1989). Because thinking about high-level and goal-related information is characteristic of abstract construals (Liberman and Trope 1998; Trope, Liberman, and Wakslak 2007), we predict that states of confidence naturally orient consumers to construe information at an abstract level. As a consequence, confidence should lead to the construction of schematic, decontextualized representations of objects and describing events in terms of the superordinate, essential, and goal-related features.

In contrast, when feeling low in confidence, people are uncertain about whether they have enough knowledge to handle the issue or whether the course of action is correct. In such a situation, people tend to focus on the "data" at hand and place their thinking toward low-level details and contextualized information (Chaiken et al. 1989; Maheswaran and Chaiken 1991; Weary and Jacobson 1997; Wood and Lynch 2002). Focusing on low-level details and incidental information in the context, as done by people with low confidence, is the characteristic of low-level and concrete construals (Liberman and Trope 1998; Trope and Liberman 2003; Trope et al. 2007). We thus predict that low confidence orients an individual to construe information at a concrete level. As a consequence, low confidence should lead to contextualized representations of objects and include subordinate, incidental, and means-related features of events (Liberman and Trope 1998; Trope and Liberman 2003; Trope et al. 2007).

Although not tested explicitly, our proposition is consis- 
tent with several findings in the literature. Research on action identification theory (Vallacher and Wegner 1987) finds that the level of confidence influences the way people identify objects. When an activity is hard to handle, due to task difficulty or lack of experience, which might be associated with low confidence, people are more likely to focus on the low-level details of objects (Vallacher and Wegner 1987; Vallacher, Wegner, and Somoza 1989). Conversely, situations that could be linked to high confidence appear to lead individuals to think more about the high-level identity of objects that signifies why the action is performed (e.g., the goal and meaning of the action). For example, Vallacher et al. (1989) found that when making a speech to audiences perceived to be relatively easy to persuade (i.e., participants could be confident), individuals focused on the high-level identity of this action, such as the significance and the goal of the speech.

Findings from the literature on positive affect are also consistent with the proposition that states of high or low confidence can produce different construals. High confidence is a characteristic often related to positive affect (Lyubomirsky, King, and Diener 2005), and positive affect has been found to increase abstract construals (Labroo and Patrick 2009). Although not tested empirically, it is possible that the confidence accompanying positive affect is involved in generating thoughts and construals at the abstract level.

Finally, prior research on power further supports the idea that high confidence might lead to abstract construals. States of high power are found to increase individuals' general sense of confidence (Briñol et al. 2007; Rucker and Galinsky 2008). Furthermore, independent research has linked a state of high power to abstract thinking. Smith and Trope (2006) found that individuals primed with high power tended to categorize stimuli at a high level of identity. The association between confidence and power and the finding that power induces abstract thinking again alludes to the possibility that high confidence might be linked with abstract construals.

These findings from distinct domains, although bearing no direct tests on the relationship between confidence and the level of construal, provide anecdotal evidence consistent with our proposed relationship. Formally, we put forth the following hypothesis.

H1: High confidence will lead consumers to construe information at an abstract level, whereas low confidence will lead consumers to construe information at a concrete level.

\section{Confidence and Information Processing: The Relevance of Messages Framed with Abstract versus Concrete Construals}

A shared finding across psychology and marketing is that consumers' depth of information processing (e.g., discrimination between weak and strong arguments) is increased when the target message is viewed as highly relevant to consumers' personal well-being, personality characteristics, or even psychological states (Aaker and Lee 2001; DeBono and Packer 1991; Tormala et al. 2008). For example, Aaker and Lee (2001) showed that participants with a dominant independent self-view (interdependent self-view) regarded a message as more relevant to them when the message implied a promotion goal (prevention goal) and that this led to a better discrimination between strong and weak arguments.

In the current work, we suggest that the relation between confidence and construal level informs what type of information is relevant to people on the basis of their state of confidence. Because people in a psychological state of low confidence orientate their thinking toward low-level details and contextualized information, we suggest that they are naturally sensitive to and focused on more concrete information. Because of this focus, when people in a state of low confidence encounter concrete information, it feels like the type of information they should attend to, and therefore it is viewed as relevant, which evokes greater attention and greater information processing. In contrast, because people in a state of high confidence construct information from a top-down angle and activate a high-level and global perspective, we suggest that they are naturally sensitive to and focused on more abstract information. Because of this alternate focus, when people in a state of high confidence encounter abstract information, it feels like the type of information they should attend to, and therefore it is viewed as relevant, which evokes greater attention and greater information processing.

In the current work, we test the relationship among confidence, construal level, and information processing by systematically varying whether the same message content is presented at a low or a high level of construal. Specifically, on the basis of the notion that information can be represented at different levels of construal (Liberman and Trope 1998; Trope and Liberman 2003), it is possible to frame a message containing the same content in either an abstract or a concrete level of construal. For example, a fitness club could be framed at a concrete level of construal by highlighting its subordinate and incidental feature of practicing daily health activities or framed at an abstract level of construal by highlighting its superordinate and essential feature of building lifelong health. On the basis of our previous discussion, a message framed at a concrete level of construal should fit with the natural orientation of those in a state of low confidence and therefore be viewed as more relevant. In contrast, a message framed at an abstract level of construal should fit with the natural orientation of those in a state of high confidence and therefore be viewed as more relevant.

On the basis of past findings that information will be processed more carefully when the target message is regarded as more relevant (DeBono and Packer 1991; Tormala et al. 2008), we propose a new type of matching wherein people in a state of low confidence will engage in greater processing of messages framed using concrete construals, whereas people in a state of high confidence will engage in 
greater processing of messages framed using abstract construals.

H2: When in a state of high confidence, consumers will view messages framed at an abstract level of construal as more relevant. In contrast, when in a state of low confidence, consumers will view messages framed at a concrete level of construal as more relevant.

H3: Because of differences in relevance, consumers in a state of high confidence will engage in greater information processing (i.e., a greater differentiation between strong and weak arguments) when a message is framed at an abstract level of construal. However, consumers in a state of low confidence will engage in greater information processing when a message is framed at a concrete level of construal.

Although our hypotheses have not received a direct test, prior research that documented a decrease in information processing for high confidence (vs. low confidence) appears to have described such messages using concrete construals. For example, Edwards (2003) instructed participants to evaluate a comprehensive exam described as enabling them to enhance grades. Describing the benefit of the exam in terms of grade improvement is a more concrete construal, whereas a more abstract construal would be to present the exam as benefiting one's intellectual or career development in the long run. As another example, Tiedens and Linton (2001) exposed participants to a message that described the attributes of a camcorder in terms of the detailed specifications, which again seems to be at the concrete construal. Of course, a limitation of past work with respect to our current hypotheses is that previous work did not systematically vary construal levels. It is therefore unclear whether the concreteness versus abstraction played a role in these studies. Thus, the link between construal frame of messages and information processing remains to be examined.

We tested our hypotheses across five experiments. Experiments 1-3 tested whether high confidence and low confidence lead to qualitatively different thinking associated with abstract and concrete construals (hypothesis 1), respectively. Experiments 4 and 5 examined how states of low versus high confidence interact with the construal frame of the message to affect perceived relevance (hypothesis 2) and information processing (hypothesis 3 ).

\section{EXPERIMENT 1: RELATING CONFIDENCE TO CONSTRUAL LEVEL}

In experiment 1 we tested our proposition that high confidence leads to an abstract construal and low confidence leads to a concrete construal. Specifically, we manipulated participants' state of confidence (low vs. high) and then measured their construal level using the Behavioral Identification Form (BIF; Vallacher and Wegner 1989), an es- tablished measure of construal level (Agrawal and Wan 2009; Fujita et al. 2006).

\section{Method}

Ninety-seven undergraduate students in Hong Kong participated in this study in exchange for HK\$60. Participants were randomly assigned to high-confidence, low-confidence, or baseline conditions. They first completed a fourquestion quiz about fitness knowledge. These questions were moderately difficult, so that participants would be able to respond but were not likely to be very confident about the accuracy of their answers. Next, we gave participants in the high- and the low-confidence conditions false feedback about their knowledge level. Participants in the high-confidence condition received feedback that they had adequate knowledge about fitness and that they could benefit by using such knowledge. In contrast, those in the low-confidence condition received feedback that their knowledge about fitness was inadequate and problematic and that they should be careful in using such knowledge. After reviewing the feedback, participants rated their confidence in fitness knowledge on a 9-point scale $(1=$ not confident at all; 9 $=$ very confident). Participants in the baseline condition did not perform the quiz task and instead wrote a short essay about their last grocery-shopping experience (Rucker and Galinsky 2008).

Next, participants were asked to complete the BIF (Vallacher and Wegner 1989). The BIF consisted of 25 questions that ask participants to read a statement of an action (e.g., making a list) and two descriptions of this action. One description defined the action in terms of how it was performed (e.g., writing something down), consistent with a concrete construal. The other description defined the action in terms of why it was performed (e.g., getting things organized), consistent with an abstract construal. For each question, participants chose the description they found to better capture the activity. In addition, participants reported their mood on five 9-point semantic differentials (sad-happy, feeling bad-feeling good, irritable-pleased, depressedcheerful, upset-joyful). Finally, participants were debriefed, paid, and thanked.

\section{Results}

Confidence Manipulation Check. A one-way ANOVA between the low- and the high-confidence conditions demonstrated that participants who received feedback that they had adequate knowledge reported feeling more confident $(M=6.26, \mathrm{SD}=1.54)$ than participants who received the feedback that their knowledge was inadequate $(M=4.15$, $\mathrm{SD}=1.56 ; F(1,65)=30.98, p<.001)$.

Construal Level. Following Vallacher and Wegner (1989), we obtained a construal level score for each participant by assigning 0 for choosing the concrete construal option and 1 for choosing the abstract construal option for each of the 25 actions in the BIF questionnaire. The scores were 
summed such that a higher score indicates a higher construal level. A one-way ANOVA indicated a significant effect of the confidence manipulation $(F(1,94)=9.56, p<.001)$. Those in the low- $(M=15.09$, SD $=3.91)$ and the highconfidence $(M=19.12, \mathrm{SD}=3.42)$ conditions differed significantly $(F(1,94)=19.12, p<.001)$. In addition, participants in the baseline condition $(M=17.10, \mathrm{SD}=$ 3.97) scored lower on the BIF than participants in the highconfidence condition $(F(1,94)=4.57, p<.04)$ but higher than those in the low-confidence condition $(F(1,94)=$ $4.47, p<.04)$. These results support hypothesis 1 that high confidence (low confidence) is associated with more (less) abstract construals.

Mood. A one-way ANOVA on a composite of the five general mood questions $(\alpha=.93)$ indicated no differences in participants' mood as a function of whether participants were primed to feel high $(M=5.89, \mathrm{SD}=1.12)$ or low confidence $(M=6.00, \mathrm{SD}=1.04)$ or were in the baseline condition $(M=5.68, \mathrm{SD}=1.08 ; F<1)$. We measured participants' mood in all experiments and found that it does not explain our results. We therefore do not discuss mood measures further.

In addition to this experiment, we replicated the results in a population of American participants. Details of this additional experiment are available from the authors.

\section{EXPERIMENT 2: THE CATEGORIZATION TASK}

Experiment 2 tested the link between confidence and construals by using a different manipulation of confidence and assessed how confidence affected the breadth of categories used in a categorization task. According to research on construal level (Liberman, Sagristano, and Trope 2002; Trope and Liberman 2003), abstract categories are more inclusive than concrete categories (e.g., the abstract category of food is more inclusive than the concrete category of dessert). If high confidence leads to an abstract construal but low confidence leads to a concrete construal, participants should generate fewer categories to classify objects when they are in a state of high versus low confidence.

\section{Method}

Fifty-one undergraduate students in Hong Kong participated in this study, and each received HK\$60. Participants were randomly assigned to a low-confidence, a high-confidence, or a baseline condition. They first performed an advertising evaluation study that was used to place participants in a relative state of high or low confidence. The cover story of this task informed participants that they would carefully review and answer questions about three advertisements for a wealth management service. High versus low confidence was manipulated by using two different sets of advertising slogans. One set of slogans highlighted the implications of being highly confident and certain, with advertising copy such as "With confidence, you can reach truly amazing heights." The other set of slogans emphasized the implications of being low in confidence, doubtful, and uncertain, with copy such as "Doubt is the beginning, not the end, of wisdom." Presenting three ads in each condition was used to prime high or low confidence among participants (for a conceptually similar idea, see Collins and Loftus 1975; Hamann 1990).

After reading the ads, participants rated how confident they felt on a 9-point scale ranging from 1 (not at all confident) to 9 (very confident). Participants then indicated their attitudes toward the ads on three 9-point semantic differentials (bad-good, negative-positive, dislike-like), with higher numbers indicating more favorable attitudes. Participants in the baseline condition did not receive the advertising study but, as in experiment 1 , were instead asked to write a short essay about a previous grocery-shopping experience.

Next, participants were asked to classify 44 objects related to camping (e.g., brush, tent, camera) into groups. They were instructed that every item should be included and there should be no overlaps between groups. Following past research (Liberman et al. 2000), we used the number of groups that participants classified as a measure of construal level (Liberman et al. 2002). Finally, participants were debriefed, paid, and thanked.

\section{Results}

Confidence Manipulation Check. A one-way ANOVA revealed a significant effect of the confidence manipulation $(F(1,49)=9.05, p<.01)$. Participants in the high-confidence condition $(M=5.94, \mathrm{SD}=1.08)$ were more confident than those in the low-confidence condition $(M=$ $4.00, \mathrm{SD}=1.46 ; F(1,49)=18.09, p<.001)$. In addition, participants in the baseline condition felt less confident ( $M=5.00, \mathrm{SD}=1.41)$ than those in the high-confidence condition $(F(1,49)=4.25, p<.05)$ but more confident than those in the low-confidence condition $(F(1,49)=$ $4.80, p<.04)$. A one-way ANOVA on a composite of the three attitude items $(\alpha=.89)$ showed that the ads used to elicit high $(M=5.54, \mathrm{SD}=1.98)$ or low confidence $(M=5.33, \mathrm{SD}=1.30)$ did not foster differences in attitudes $(F<1)$. Similar null effects on attitudes were found in the other studies using the advertising task to manipulate confidence, and we will not discuss this measure further.

Number of Groups Categorized. A one-way ANOVA on the number of groups classified by participants indicated a significant effect $(F(1,49)=8.88, p=.001)$. Participants in the baseline condition classified the items for camping into more groups $(M=5.35, \mathrm{SD}=1.83)$ than those in the high-confidence condition $(M=4.17, \mathrm{SD}=1.33 ; F(1,49)=$ 4.03, $p=.05$ ) but into fewer groups than those in the lowconfidence condition $(M=6.65, \mathrm{SD}=1.90 ; F(1,49)=$ $4.87, p<.04)$. In addition, the difference between the lowand the high-confidence conditions was significant $(F(1,49)$ 
$=17.75, p<.001)$. These results further support hypothesis 1 that states of high confidence trigger a focus on abstract construals, whereas states of low confidence trigger a focus on concrete construals.

\section{EXPERIMENT 3: THE TRADE-OFF BETWEEN FEASIBILITY AND DESIRABILITY}

Experiment 3 used a third approach to examine the link between states of high versus low confidence and construal level in a consumer decision context. We manipulated states of high versus low confidence and observed participants' preference for choice options involving the trade-off between the desirability and feasibility. Desirability is associated with more abstract construals, and feasibility, with more concrete construals (Liberman and Trope 1998; Trope and Liberman 2003). If high confidence leads to an abstract level of construal, high-confidence participants should exhibit a greater preference for desirability than do low-confidence participants.

\section{Method}

Sixty-one undergraduate students in Hong Kong participated in this study in exchange for HK\$60. Participants first completed the advertising study to manipulate high versus low confidence and then rated their confidence as in experiment 2. Next, participants completed a choice task adapted from Liberman and Trope (1998, experiment 2). The cover story had participants imagine they were required to attend a guest lecture at their university. Participants were told they had to attend one of two lectures that varied with respect to whether the lecture topic was interesting and relevant (i.e., desirability-the aspect associated with an abstract construal) or whether the lecture schedule was convenient (i.e., feasibility - the aspect associated with a concrete construal). The lecture with high desirability and low feasibility was described as more interesting but took place early in the morning on a day when students did not have to go to school. The lecture with low desirability and high feasibility was described as less interesting but occurred at a time when the students were already at school. After reading the descriptions, participants indicated their choice. Finally, participants were debriefed, paid, and thanked.

\section{Results and Discussion}

Confidence Manipulation Check. A one-way ANOVA confirmed that participants in the high-confidence condition reported feeling more confident $(M=6.39, \mathrm{SD}=1.32)$ than participants in the low-confidence condition $(M=$ $5.16, \mathrm{SD}=1.50 ; F(1,60)=11.63, p=.001)$.

Choice of Guest Lecture. We first performed a binary logistic regression using the confidence states as the independent variable $(1=$ high confidence; $0=$ low confidence $)$ and choice as the dependent variable $(1=$ chose the high- desirability option; $0=$ chose the high-feasibility option). The results indicated that being more confident led participants to be more likely to choose the high-desirability option $(\beta=1.47$, Wald test $=3.98, p<.05)$. We then compared the choice share of the lectures across conditions. For participants in the high-confident condition $(n=33)$, a larger proportion of them $(91.9 \%)$ chose to sign up for the lecture with high desirability over the lecture with high feasibility $(9.1 \% ; z=6.40, p<.001)$. The reverse was found for less confident participants $(n=28)$, with a larger proportion of them choosing to sign up for the lecture with high feasibility $(71.4 \%)$ compared to the one with high desirability $(28.6 \% ; z=2.94, p<.05)$.

From another perspective, a larger proportion of participants in the high-confident condition, compared to the lowconfident condition, chose the lecture with high desirability $(z=4.87, p<.001)$, whereas a larger proportion of participants in the low-confident condition, compared to the highconfident condition, chose the lecture with high feasibility $(z=4.79, p<.001)$. These results further support that high confidence (vs. low confidence) led participants to construe at the abstract level (hypothesis 1) and consequently increased the preferences for the choice option with high desirability compared to the choice option with high feasibility.

Using three distinct approaches in experiments 1-3, with independently recognized measures from the construal literature and different manipulations of high versus low confidence, we found converging support for our first hypothesis that high confidence leads people to focus on more abstract construals, whereas low confidence leads people to focus on more concrete construals. In the next set of experiments, we examined how confidence influences participants' information processing in a product evaluation context in relation to messages framed in an abstract versus concrete fashion.

\section{EXPERIMENT 4: ALIGNING CONFIDENCE WITH CONSTRUAL FRAME}

Given that experiments 1-3 demonstrated that a state of low versus high confidence appears to naturally orient people toward a low versus high level of construal, we predicted that this would have implications for what type of information naturally fits with their state. In accordance with our second hypothesis, we predicted that a message framed in an abstract fashion (e.g., the message is presented as highlighting essential and goal-related features of an issue or object) would naturally fit with the orientation of a state of high confidence, leading such messages to be viewed as more relevant. Conversely, messages framed in a concrete fashion (e.g., the message is presented as highlighting incidental and means-related features of an issue or object) would naturally fit with the orientation of a state of low confidence, leading such messages to be viewed as more relevant. As a consequence of differences in perceived relevance, individuals in a state of low confidence should process a message framed at a concrete level of construal more 
deeply, whereas individuals in a state of high confidence should process a message framed at an abstract level of construal more deeply.

To test our predictions, we manipulated participants' confidence, exposed them to product messages framed using either abstract or concrete construals, manipulated the message arguments to be either strong or weak, and assessed the perceived relevance of the message. The inclusion of an argument quality manipulation, strong versus weak, is an established measure of depth of information processing (Aaker and Lee 2001; Petty and Wegener 1998; Wan et al. 2010). Attitudinal differences between strong- and weakargument conditions are known to be larger when individuals process information carefully. Therefore, according to our perspective, attitudinal differences between strong and weak arguments should be greater for participants in a state of high confidence (low confidence) when the message is framed in an abstract construal (a concrete construal).

The argument quality manipulation also allows us to address an alternative explanation for the proposed effect. One could argue that the matching between one's state of confidence and the message frame in an abstract construal (a concrete construal) might induce a positive experience due to processing ease (Wänke, Bless, and Biller 1996), and this positive experience might be used as a simple cue to enhance individuals' attitudes toward the described object, regardless of the quality of the message. Unlike an information-processing perspective, a simple cue explanation predicts a main effect of matching on attitudes rather than an interaction involving greater discrimination of argument quality.

Of course, it is also possible that differences in ease could explain our results to the extent that a match between a state of high (low) confidence and an abstract (concrete) message frame makes it easier for individuals to process the message and thus orients them to process it more. That is, a difference in depth of information processing might not be driven by information being viewed as more relevant but by information being easier to process. To address this possibility, we measured processing ease in the current experiment.

Experiment 4 manipulated the abstract versus concrete construals activated in the message frame by describing the benefit of the target object in a goal-related frame or a means-related frame (Liberman and Trope 1998; Trope and Liberman 2003). For example, for the event of moving to a new apartment, thinking about the goal and why to move (e.g., to start a new life) is consistent with an abstract construal. In contrast, thinking about the means of moving and how to carry out the move (e.g., packing and carrying boxes) is consistent with a concrete construal (Liberman and Trope 1998). Importantly, we introduced the abstract versus concrete frames at the outset of the processing task and kept the entire body of the message identical across construal conditions. This approach allowed us to ensure that if we observed differences in processing depth and attitudes, we could attribute these differences solely to participants' state of confidence and the construal framing of the message as opposed to any differences or weighting of the type of arguments within the message content itself.

\section{Method}

One hundred and sixty-one undergraduate students in Hong Kong participated in this study. Each of them received $\mathrm{HK} \$ 60$. Participants were randomly assigned to one of 12 conditions in a 3 (confidence state: high vs. low vs. baseline) $\times 2$ (message frame: abstract construal vs. concrete construal) $\times 2$ (argument quality: strong vs. weak) betweensubjects design. First, participants completed the advertising study that primed high versus low confidence and the same manipulation check questions as used in experiments 2 and 3. Participants in the baseline condition did not receive this manipulation.

Next, participants were asked to evaluate a fitness club. They were first presented with a slogan for promoting this fitness club, which actually manipulated the message frame to be abstract or concrete. In both the abstract frame and the concrete frame conditions, the slogan highlighted the benefit of joining this fitness club to health. Adopting the method used in Labroo and Patrick (2009, experiment 2b), we described the benefit to health in either an abstract and future-oriented manner (i.e., "enjoy lifelong health") or a concrete and present-oriented manner (i.e., "enjoy day-today health activities"). A pretest with participants from the same participant pool established the effectiveness of this manipulation.

After reading the slogan, participants moved to another page to read about the major facilities available in the target fitness club. In the strong-arguments condition, participants read that the fitness club offered a full range of exercise facilities, individualized exercise classes on a regular basis, and a personal trainer service on a daily basis. In the weakarguments condition, participants read that this fitness club offered a limited range of exercise facilities, individualized exercise classes on special request, and a personal trainer service on the weekend. We pretested the strong and the weak arguments using a separate sample of 40 participants from the same participant pool, which established that, although the messages for the fitness club described favorable features in general, they differed in persuasiveness.

After reading the message, participants reported their attitudes on four 9-point semantic differentials (bad-good, negative-positive, unfavorable-favorable, dislike-like), with higher numbers indicating more favorable attitudes. In addition, participants responded to three questions that measured their perceived relevance of the message: How relevant was the message about the fitness club to you/to your values/to your interest? Participants also answered two questions about the ease of processing: How difficult (reverse coded)/smooth was it to process the message? Responses to these questions were all anchored on scales of 1 (not at all) to 9 (very much). Finally, participants were debriefed, paid, and thanked. 


\section{Results and Discussion}

Confidence Manipulation Check. A $2 \times 2 \times 2$ ANOVA (without the baseline conditions) on participants' confidence indicated only a significant main effect of the confidence manipulation: participants in the high-confidence condition reported greater confidence $(M=5.52, \mathrm{SD}=1.50)$ than participants in the low-confidence condition $(M=4.31$, $\mathrm{SD}=1.44, F(1,114)=19.89, p<.001)$. No other effects were significant (all $F<1$ ).

Attitudes toward the Fitness Club. We averaged the attitude items to form a fitness club attitude index $(\alpha=.96)$. A $3 \times 2 \times 2$ ANOVA resulted in a significant main effect of argument quality $(F(1,149)=48.51, p<.001)$, a marginally significant argument quality $\times$ frame interaction $(F(1,149)=2.80, p=.064)$, and a significant three-way interaction on attitudes $(F(2,149)=13.89, p<.001$; see fig. 1). No other effects were significant (all $p>.60$ ). We decomposed this three-way interaction into two separate two-way interactions to better understand the nature of this interaction.

When the message framed the benefit of joining the fitness club in a concrete construal (enjoy daily health activity), there was a significant confidence state $\times$ argument quality interaction $(F(2,149)=8.61, p<.001)$. For participants primed to feel low in confidence, they had more favorable attitudes toward the fitness club when the arguments were strong $(M=6.13, \mathrm{SD}=1.07)$ than when they were weak $(M=3.28, \mathrm{SD}=1.28 ; F(1,149)=42.68, p<.001)$. However, for participants primed to feel highly confident, they did not differ in their attitudes whether the arguments were strong $(M=5.05, \mathrm{SD}=.98)$ or weak $(M=4.62, \mathrm{SD}=$ $1.46 ; F<1)$. Moreover, the attitudes of participants in the baseline condition were similar to those in the high-confidence condition and did not differentiate between strong$(M=5.25, \quad \mathrm{SD}=.86)$ and weak-argument conditions $(M=4.47, \mathrm{SD}=1.21 ; F(1,149)=2.02, p>.15)$. These results replicate the classic finding that less confidence is associated with more information processing.
When the message framed the benefit of joining the fitness club in an abstract construal (enjoy lifelong health), there was also a significant confidence state $\times$ argument quality interaction $(F(2,149)=8.11, p<.001)$ but with a very different form. Participants induced to feel highly confident had more favorable attitudes when the arguments were strong $(M=6.26, \mathrm{SD}=1.13)$ than when they were weak $(M=3.48, \mathrm{SD}=1.53 ; F(1,149)=42.50, p<.001)$. However, participants induced to feel less confident did not differentiate their attitudes between the strong- $(M=5.14$, $\mathrm{SD}=1.31)$ and the weak-argument conditions $(M=$ $4.48, \mathrm{SD}=1.13 ; F(1,149)=2.36, p>.12)$. Moreover, participants in the baseline condition were similar to less confident participants in attitudes and did not differentiate between the strong- $(M=5.27, \mathrm{SD}=.93)$ and the weakargument conditions $(M=4.75, \mathrm{SD}=1.11 ; F<1)$. These results demonstrate a reversal of the classic informationprocessing effect. In addition, the results in the baseline condition showed that their attitudes were similar to those in conditions in which there was a mismatch between the confidence state and the construal frame, which suggests that the enhanced or reduced attitudes favorability was driven by the conditions in which the construal frame enhanced the message relevance in the state of high or low confidence.

Relevance as the Mediator. Participants' responses to the three questions that measured their perceived relevance of the message were averaged to form a relevance score $(\alpha=.80)$. Next, a $3 \times 2 \times 2$ ANOVA on the relevance score indicated a marginally significant main effect of confidence state $(F(2,149)=2.92, p=.058)$ and, importantly, a significant confidence state $\times$ construal frame twoway interaction $(F(2,149)=12.45, p<.001)$. No other effects were significant (all $F<1$ ). Simple contrasts showed that highly confident participants perceived the message as more relevant to them when it was framed in the abstract construal $(M=5.57, \mathrm{SD}=1.22)$ than in the concrete construal $(M=4.55, \mathrm{SD}=1.19 ; F(1,149)=10.81, p<.01)$,

\section{FIGURE 1}

RESULTS FOR FITNESS CLUB ATTITUDES (EXPERIMENT 4)

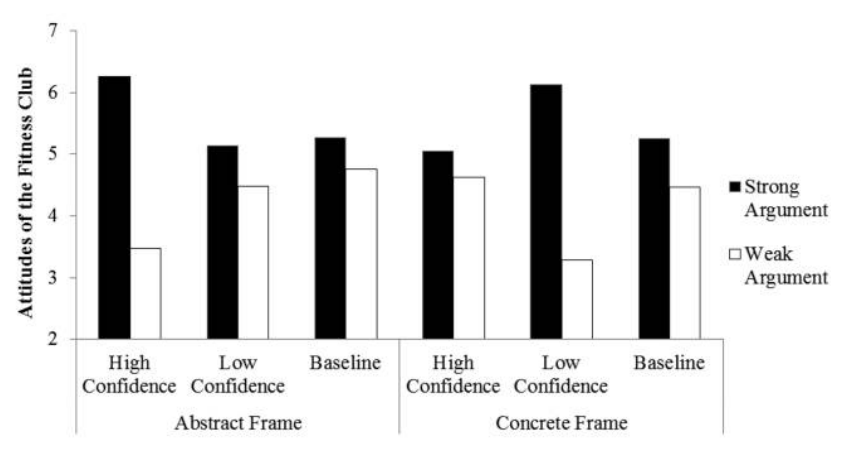


whereas the opposite was true for less confident participants: they perceived the message as more relevant when it was framed in the concrete construal $(M=5.50, \mathrm{SD}=1.26)$ than in the abstract construal $(M=4.36, \mathrm{SD}=1.29$; $F(1,149)=13.44, p<.001)$. Participants in the baseline condition did not differ in their perceived relevance of the message, whether the message was framed in the concrete $(M=4.32, \mathrm{SD}=1.05)$ or abstract construal $(M=4.65$, $\mathrm{SD}=.95 ; F<1)$. Regardless of argument quality, participants considered the message as more relevant to them when high confidence (low confidence) was aligned with abstract (concrete) construals. Moreover, consistent with the results on attitudes, participants in the baseline condition had similar perceived message relevance to those in conditions in which the confidence states did not match the construal frame for the message.

Next, we examined the mediating role of relevance by performing a mediated moderation analysis using the procedures recommended by Preacher, Rucker, and Hayes (2007). In this analysis we excluded the baseline condition to focus on examining the process underlying the matching (vs. mismatching) between participants' state of confidence and construal frame. Because the confidence state $\times$ message frame effects on fitness club attitudes were opposite in the strong- and the weak-argument conditions, we followed the analysis procedures used in the literature (Briñol et al. 2007) and reverse coded the attitude score in the weakargument condition to create an "adjusted attitude" score before conducting the mediation analysis. This procedure essentially creates a measure of the extent of information processing, by equating larger scores with greater information processing.

All independent variables were standardized before being submitted to the following analyses. First, regressing the adjusted attitude score on confidence state $(1=$ high confidence; $0=$ low confidence), frame ( $1=$ abstract construal; $0=$ concrete construal), and their interaction indicated a significant interaction effect $(\beta=.42, t(1,118)=5.05$, $p<.001)$. Next, regressing the relevance score on confidence state, frame, and their interaction produced a significant interaction effect $(\beta=.40, t(1,118)=4.78, p<$ $.001)$. Finally, we regressed the adjusted attitude score on confidence state, frame, confidence state $\times$ frame interaction, and relevance. The results showed that the effect of relevance on attitudes was significant $(\beta=.55, t(1,117)=$ $7.12, p<.001)$, and the size of the confidence state $\times$ frame interaction effect on attitudes was clearly reduced $(\beta=$ $.20, t(1,117)=2.64, p<.01$; see fig. 2$)$. Finally, the results of a $95 \%$ confidence interval indicated that the indirect effect was significantly different from zero $(95 \% \mathrm{CI}=.16$ to .45$)$, which provided evidence of mediation (Preacher et al. 2007; Shrout and Bolger 2002).

We followed the same procedure to test the role of processing ease. Participants' responses to the two ease-of-processing questions were averaged $(r=.69, \alpha=.81)$. A 3 $\times 2 \times 2$ ANOVA indicated no significant effects of experimental treatment on the ease of processing (all $p>$
FIGURE 2

PERCEIVED MESSAGE RELEVANCE AS THE MEDIATOR (EXPERIMENT 4)

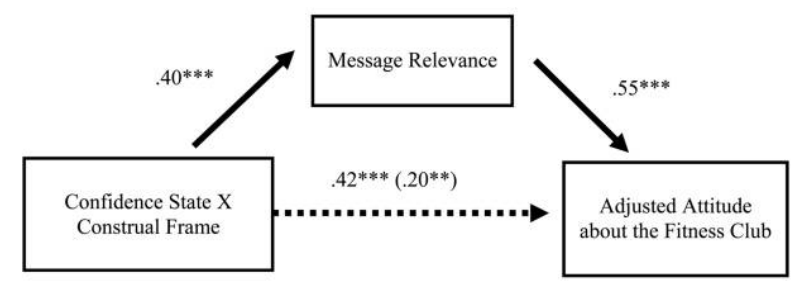

NOTE._ ${ }^{\star \star}=$ significant at .01 level; ${ }^{\star \star \star}=$ significant at .001 level.

.14). Thus, perceived ease or difficulty in message processing could not account for the results. We found similar results on ease in experiment 5 and will not discuss this variable further.

Discussion. Findings from experiment 4 support hypotheses 2 and 3. A frame that focuses individuals on abstract versus concrete levels of construal has implications for how relevant a message is perceived to be by individuals in a state of high versus low confidence (hypothesis 2), and this in turn affects the depth of their information processing of the message (hypothesis 3).

Experiment 4 also ruled out an alternative possibility relating to experienced ease fostering a positive experience that influences individuals' attitudes. First, there was no observed effect on participants' self-reported ease. Second, presenting participants in a state of high confidence (low confidence) with a message framed in an abstract (a concrete) manner enhanced attitude favorability when message arguments were strong but reduced attitude favorability when message arguments were weak. If the underlying process was not relevance but simply a positive experience associated with ease as a simple heuristic, then we should have observed a main effect across argument quality conditions, which we did not. Moreover, the null effects on processing ease also suggest that the difference in the depth of information processing was not driven by differences in fluency occurring as a function of confidence and construal framing.

\section{EXPERIMENT 5}

Experiment 5 aimed to increase the generalizability of our findings. First, experiment 5 manipulated confidence using a recall task adopted from past research (Gal and Rucker 2011; Tormala et al. 2008). Second, experiment 5 manipulated the message frame associated with construals on the basis of the well-documented finding that thinking about the distant future leads to an abstract level of construal and thinking about the near future leads to a concrete level of 
construal (Liberman et al. 2002; Nussbaum, Trope, and Liberman 2003). Thus, in experiment 5 we varied the message frame by describing the event in the message with temporal distance varied. As in experiment 4, we introduced the abstract versus concrete frames at the outset of the processing task and kept the actual messages identical.

Experiment 5 also added two new dependent measures in addition to the attitude measures, one assessing behavioral intention and the other examining message-related thoughts. Finally, experiment 5 tested the matching effect in a new context.

\section{Method}

One-hundred and twelve undergraduate students in Hong Kong participated in this study. Each of them received $\mathrm{HK} \$ 60$. Participants were randomly assigned to one of eight conditions in a 2 (confidence state: high vs. low) $\times 2$ (message frame: abstract construal [distant future] vs. concrete construal [near future]) $\times 2$ (argument quality: strong vs. weak) between-subjects design. Participants first completed an episodic recall task adopted from past research to manipulate high versus low confidence (Gal and Rucker 2011; Tormala et al. 2008). Specifically, participants were told that they should put themselves in a state of reliving past experiences and were asked to write down those experiences. Participants in the high-confidence condition were asked to recall and list four experiences in which they felt highly confident and certain, and those in the low-confidence condition were asked to recall four experiences in which they felt doubtful and uncertain. Upon completing the recall task, participants rated their feeling of confidence on a scale of 1 (not confident at all) to 9 (very confident).

Next, as part of an ostensibly unrelated task, participants were asked to review information about a hotel. Participants completed the task in two parts. The first part manipulated the frame associated with construals, by telling participants to imagine that they were booking a hotel for a vacation trip next year (distant future) in the abstract construal condition or that they were booking a hotel for a vacation trip next week (near future) in the concrete construal condition. The effectiveness of this manipulation was successfully confirmed in a pretest.

The second part of the task presented a message about the target hotel in the form of customer reviews. In the strong-argument condition, the reviews commented on this hotel as having highly impressive decor with valuable paintings, excellent food with a wide range of choices in its restaurant, friendly staff fluent in English, clean rooms with high-quality furniture, highly flexible room service, and so on. In the weak-argument condition, the reviews depicted this hotel as having fine but less impressive decorations using prints, nice foods with limited choices in its restaurant, friendly staff but with poor English, reasonably clean rooms and basic furniture, regular room service without much flexibility, and so on. We pretested the strong and weak arguments using a separate sample of participants from the same participant pool, which established that, although the cus- tomer reviews in general talked about favorable things about this hotel, the arguments in the two conditions differed in persuasiveness when scrutinized carefully.

After reading the messages about the hotel, participants indicated their attitudes on the same four 9-point semantic differentials used in experiment 4 , with higher numbers indicating more favorable attitudes. Then, participants rated the likelihood that they would book this hotel on a scale of 1 (not at all likely) to 9 (very likely) and completed the same measures as used in experiment 4 to assess the perceived relevance of the message and ease of processing. Moreover, participants completed a thought-listing procedure adopted from past research, a measure highly sensitive to processing differences (Petty and Wegener 1998; Rucker and Petty 2006). Finally, participants were debriefed, paid, and thanked.

\section{Results and Discussion}

Confidence Manipulation Check. A $2 \times 2 \times 2$ ANOVA performed on participants' self-reported confidence revealed only a significant main effect of the confidence manipulation: participants who recalled experiences about high confidence reported feeling more confident $(M=6.34$, SD = 1.51) than did participants who recalled experiences about low confidence $(M=3.88, \mathrm{SD}=1.37 ; F(1,104)=80.28$, $p<.001$ ). No other effects were significant (all $p>.15$ ).

Attitude and Behavioral Intention about the Hotel. Participants' responses to the four attitude items were averaged to form an attitude index $(\alpha=.97)$. A $2 \times 2 \times 2$ ANOVA resulted in a significant main effect of argument quality $(F(1,104)=220.81, p<.001)$ and a significant three-way interaction on the attitudes toward the hotel $(F(1,104)=20.66, p<.001$; see fig. 3). All other effects were not significant (all $p>.72$ ).

Decomposing the three-way interaction into separate twoway interactions, we found that when the hotel message was framed in terms of a concrete construal (planning for a trip next week), there was a significant confidence state $\times$ argument quality interaction $(F(1,104)=11.96, p<.001)$.

\section{FIGURE 3}

\section{RESULTS FOR HOTEL ATTITUDES (EXPERIMENT 5)}

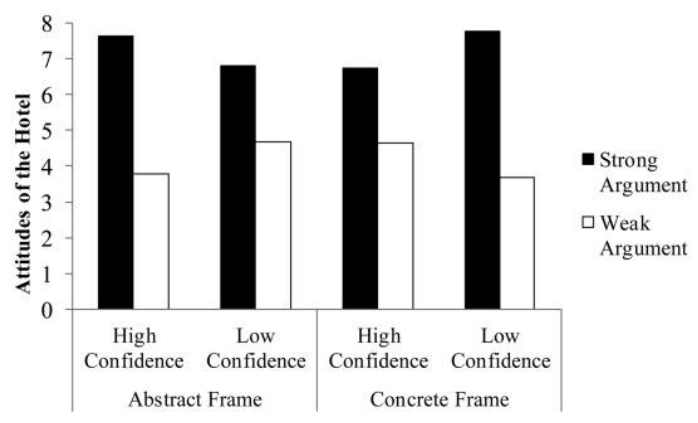


This interaction revealed that participants in a state of low confidence exhibited a greater difference between strong $(M=7.77, \mathrm{SD}=.93)$ and weak arguments $(M=3.67$, $\mathrm{SD}=1.27 ; F(1,104)=100.03, p<.001)$ compared to participants in a state of high confidence (strong: $M=6.73$, $\mathrm{SD}=1.14$; weak: $M=4.64, \mathrm{SD}=1.05 ; F(1,104)=$ $26.11, p<.001)$. These findings replicate the classic effect in the literature that low confidence is associated with greater information processing compared with high confidence.

When the hotel message was framed in terms of an abstract construal (planning for a trip next year), there was also a significant confidence state $x$ argument quality interaction $(F(1,104)=8.78, p<.01)$. However, this twoway interaction took the opposite form of that found when the message was framed in a concrete construal. High-confidence participants exhibited a stronger differentiation between strong $(M=7.63, \mathrm{SD}=1.05)$ and weak arguments $(M=3.78, \mathrm{SD}=.96 ; F(1,104)=88.18, p<.001) \mathrm{com}-$ pared to low-confidence participants (strong: $M=6.82$, $\mathrm{SD}=.97 ;$ weak: $M=4.69, \quad \mathrm{SD}=1.19 ; \quad F(1,104)=$ $27.01, p<.001)$. These results reverse the classic relationship between confidence state and information processing.

Similar analyses were conducted to examine participants' intention to book the hotel. The $2 \times 2 \times 2$ ANOVA indicated a significant main effect of argument quality $(F(1,104)=$ $126.67, p<.001)$ and a significant three-way interactions on intention to book a room at the hotel $(F(1,104)=24.69$, $p<.001$ ). All other effects were not significant (all $p>$ .38). Decomposing the three-way interaction into two separate two-way interactions revealed similar patterns as those on the attitudes measure. See table 1 for means and significance tests across conditions.

Thoughts. Participants' thoughts related to the hotel message were classified as positive, negative, or neutral by two judges unaware of the conditions and hypotheses. Judges agreed on $97 \%$ of the thought classifications, and disagreements were resolved by discussion. To form a thought favorability index, we subtracted the number of negative message-related thoughts from the number of positive message-related thoughts and divided this difference by the total number of message-related thoughts (Cacioppo and Petty 1981). The results on the thought favorability mirrored the results on the attitudes about the hotel (see table 1).
Relevance as the Mediator. Participants' responses to the three questions that measured their perceived relevance of the message were averaged to form a relevance score $(\alpha=.88)$. A $2 \times 2 \times 2$ ANOVA on the relevance score indicated a significant confidence state $\times$ message frame two-way interaction $(F(1,104)=22.88, p<.001)$. No other effects were significant (all $p>.14$ ). Simple contrasts showed that participants in a state of high confidence perceived the message as more relevant to them when it was framed in the abstract construal $(M=6.48, \mathrm{SD}=1.38)$ than in the concrete construal $(M=5.42, \mathrm{SD}=1.41$; $F(1,104)=9.08, p<.01)$, whereas participants in a state of low confidence perceived the message as more relevant when it was framed in the concrete $(M=6.61, \mathrm{SD}=$ 1.14) rather than the abstract construal $(M=5.29, \mathrm{SD}=$ $1.26 ; F(1,104)=14.13, p<.001)$.

Next, we examined the mediating role of relevance by performing a mediated moderation analysis using the same procedure described in experiment 4 . Regressing the adjusted attitude score on confidence state, frame, and their interaction indicated a significant interaction effect $(\beta=$ $.30, t(1,108)=3.81, p<.001)$. Regressing the relevance score on confidence state, frame, and their interaction resulted in a significant interaction effect $(\beta=.43, t(1,108)=4.88$, $p<.001)$. Finally, regressing the adjusted attitude score on confidence state, frame, confidence state $\times$ frame interaction, and relevance showed that the main effect of relevance was significant $(\beta=.38, t(1,106)=4.03, p<.001)$, and the size of the confidence state $\times$ frame interaction effect on attitudes was clearly reduced $(\beta=.18, t(1,106)=$ $1.95, p=.052)$. A confidence interval test revealed that the indirect effect was significantly different from zero $(95 \%$ $\mathrm{CI}=.10$ to .37 ; Preacher et al. 2007; Shrout and Bolger 2002), provided evidence of mediation.

Discussion. Experiment 5 replicated the matching effect documented in experiment 4 in a different product category, used a different manipulation of confidence, and varied the framing associated with construals. Results on attitudes, thoughts, and behavioral intention showed that when the message was framed in a concrete construal, we again replicated the classic effects of confidence on information processing. However, when the message was framed in an abstract construal, these classic findings reversed: individuals

TABLE 1

RESULTS ON THE BEHAVIOR INTENTION AND THOUGHT FAVORABLENESS ABOUT THE HOTEL IN EXPERIMENT 5

\begin{tabular}{|c|c|c|c|c|}
\hline & \multicolumn{2}{|c|}{ Concrete construal frame } & \multicolumn{2}{|c|}{ Abstract construal frame } \\
\hline & High confidence & Low confidence & High confidence & Low confidence \\
\hline \multicolumn{5}{|l|}{ Behavior intention: } \\
\hline Strong argument & $6.14(1.46)$ & $7.29(1.26)$ & $7.14(1.23)$ & $6.15(1.01)$ \\
\hline Weak argument & $4.86(1.40)$ & $3.14(1.35)$ & $3.36(1.01)$ & $4.36(1.44)$ \\
\hline \multicolumn{5}{|l|}{ Thought favorability: } \\
\hline Strong argument & $.54(.53)$ & $.85(.28)$ & $.82(.28)$ & $.53(.42)$ \\
\hline Weak argument & $-.25(.53)$ & $-.65(.32)$ & $-.67(.35)$ & $-.31(.59)$ \\
\hline
\end{tabular}

NoTE.-For both the results on behavior intention and those on thought favorability, the 12 pairs of comparisons in the confidence state $x$ construal frame $\times$ argument quality interactions are all significant (all $p<.05$ ). Standard deviation in parentheses. 
in a state of high confidence processed information more than those in a state of low confidence. The mediation analysis supported that the effect was driven by differences in perceived relevance of the message.

\section{GENERAL DISCUSSION}

Traditional research on information processing has found that a state of high confidence often reduces the depth of information processing compared to a state of low confidence (e.g., Chaiken et al. 1989). The current research offers a new theoretical perspective that high- and low-confidence states lead to qualitatively different thinking captured by differences in abstract versus concrete construals. As such, we argued that the relevance of the message, and thus the depth of information processing, depends on the construal level at which the message is framed.

Support for the major propositions was found across five experiments. Experiments 1-3, using both construal-level measures and behavioral indicators in decision contexts, found that participants in a state of high confidence (low confidence) exhibited behavior more consistent with an abstract (concrete) level of construal. Experiments 4 and 5 demonstrated that whether a state of high confidence or low confidence increases or decreases information processing depends on whether a message is framed in a concrete or an abstract construal. The classic pattern of how confidence influences information processing occurred when messages were framed in concrete terms, but this pattern reversed when messages were framed in abstract terms. Furthermore, our effects were mediated by perceived relevance (experiments 4 and 5).

\section{Theoretical Contribution}

For the information-processing literature, we provide a new revelation about the psychological consequences of being in a state of high versus low confidence for subsequent information processing. A large body of prior research has found that states of high versus low confidence induce different perceptions of the self-assessed knowledge sufficiency, and such a difference leads to greater processing when consumers have low confidence as opposed to high confidence (Chaiken et al. 1989; Maheswaran and Chaiken 1991; Tiedens and Linton 2001; Weary and Jacobson 1997). The current research identifies that states of confidence can differ in the levels of construal they provoke. As a consequence, a state of high confidence can enhance subsequent information processing when the message is framed in an abstract construal (vs. a concrete construal), whereas a state of low confidence enhances subsequent information processing when the message is framed in a concrete construal (vs. an abstract construal).

In the current investigation, we focus on situations in which the confidence was incidental to the information processed. Our findings demonstrate that incidentally experienced confidence can carry over and influence the processing of messages unrelated to the context that induced a state of high or low confidence. However, in one experiment not reported in this article, we found evidence that similar effects occur when the confidence is relevant to the message domain. Specifically, we followed a procedure similar to experiment 4 , but we manipulated whether individuals felt low or high in confidence in the domain of fitness and then exposed them to a message related to fitness. Conceptually replicating experiment 4 and 5, we found that individuals with low confidence in their fitness knowledge processed a message more when it was framed with a concrete construal, whereas individuals with high confidence in their fitness knowledge processed a message more when it was framed with an abstract construal.

The current research also potentially enhances our understanding of past findings documented in the literature that high confidence (low confidence) decreases (increases) the depth of information processing (Edwards 2003; Tiedens and Linton 2001; Weary and Jacobson 1997). As discussed earlier, it is possible that in prior investigations the target message was described in relatively concrete construals (e.g., describing a comprehensive exam in terms of its lowlevel benefits in enhancing grades rather than the high-level benefits for intellectual and career development; Edwards 2003). This observation suggests that manipulating construal level might significantly moderate past findings in the literature.

The current research also extends the literature on construal levels (Liberman and Trope 1998; Trope and Liberman 2003) on two grounds. First, we identify confidence as a new psychological state that can influence construal levels. The distinction between concrete and abstract construals has played a central role in research ranging from person perception (Gilbert and Malone 1995) to social categorization (Nussbaum et al. 2003). Construal-level theory (Trope and Liberman 2003) has suggested that the abstract, high-level construal often promotes high confidence because the abstract construal contains less incidental and contextual features that have been found to undermine confidence in predictions (Griffin, Dunning, and Ross 1990; Sherman 1980). We document that the reversed causal path can also hold.

Second, our results extend recent work by Zhao and Xie (2011). Zhao and Xie find that congruity between the social distance dimension (e.g., product recommendation by close others vs. distant others) and the temporal distance dimension of a message (e.g., the product is for the use in the near future vs. distant future) increases persuasion. Furthermore, they suggest that this occurs due to a shift in perceived relevance. The current research examines the match between a new set of constructs related to construal levels and, importantly, demonstrates that the match influences the depth of information processing, which could increase or decrease attitude favorableness, depending on the quality of the accompanying arguments.

\section{Future Directions}

It will be important for future research to examine boundary conditions for the links among states of confidence, 
construals, and information processing. As one potential boundary condition, we posit that the perception of how benign or problematic the situation is might moderate the effect. We have argued that low confidence leads to the perception of having insufficient knowledge in a specific context, which indicates that individuals might be in a problematic situation. The way to address this problematic situation is to focus on the detailed information in the context, which activates concrete construals. However, if individuals perceive a situation as problematic, they should generate more concrete construals regardless of their confidence level. In an initial test of this idea, we found that when participants were primed to perceive a situation as problematic, both states of high and low confidence led participants to generate relatively concrete construals. Further studies are required to systematically examine this effect and other boundary conditions.

Demonstrating the link between confidence and construal levels also suggests an avenue for future research, which is to examine the impact of confidence on a wide range of consumer decisions. If states of high and low confidence activate different construal levels, then psychological confidence has the potential to influence consumer behavior in a variety of ways such as consumers' predictions about time (Trope and Liberman 2003), self-control (Agrawal and Wan 2009; Fujita et al. 2006), response to brand extensions (Alexander, Lynch, and Wang 2008), and processing pictorial or verbal information (Amit, Algom, and Trope 2009). Indeed, experiments 2 and 3 are examples of new effects of confidence based on recognizing its relation to construal levels. As a concrete example for future research, prior research has shown that information processing is more efficient when the information presentation is pictorial (verbal) for objects portrayed with psychological proximity (distance). Given that a state of high confidence activates abstract construals, which are associated with psychological distance, then one can predict that information processing might be enhanced for consumers in a state of high confidence when the information is presented in words rather than in pictures. Future studies can examine this prediction and its implication for advertising. 


\section{APPENDIX}

The Stimuli for Priming the State of High versus Low Confidence (Experiments 2, 3, 4)

\section{Message copy in the high confidence prime condition (one per screen):}

1. "Confidence: With confidence, you can reach truly amazing heights."

2. "Confidence: Experience tells you what to do; Confidence allows you to do it."

3. "Confidence: You can, you can, \& you can; Success comes in cans!"
Message copy in the low confidence prime condition (one per screen):

1. "Doubt: The only thing that makes life possible is permanent uncertainty."

2. "Doubt: Doubt is the beginning not the end of wisdom."

3. "Doubt: In the battle between you and the world, bet on the world."

The Frame and Message in Experiment 4

\section{Frame}

(Abstract versus Concrete Frames)

Abstract Frame:

"Enjoy life-long health!"

Concrete Frame:

"Enjoy day-to-day health activities!"
Message about the Fitness Club

(Strong versus Weak Arguments)

Strong Argument:

Major Facility Introduction:

- Equipment: Spinning studio and climbing walls; Cardio Equipment (e.g., Treadmills, Recumbent Bicycles, Elliptical, Rower); Strength Training Equipment

- Group classes with regular schedule: Yoga, Pilate, Ballet, Belly dance, Latin dance, Meditation, Body pump, abs/back/stretch, cardio box/conditioning, strength, muscle training

- Personal trainer service available 7 days a week

Weak Argument:

Major Facility Introduction:

- Equipment: Cardio Equipment, mainly Treadmills; Strength Training Equipment

- Some group classes (e.g., Yoga) can be scheduled upon request. The class types and the schedules may not be ensured. All depend on the availability of instructors.

- Personal trainer service is available on the weekend

\section{REFERENCES}

Aaker, Jennifer L., and Angela Y. Lee (2001), “'I' Seek Pleasures and 'We' Avoid Pains: The Role of Self-Regulatory Goals in Information Processing and Persuasion," Journal of Consumer Research, 28 (June), 33-49.

Agrawal, Nidhi, and Echo Wen Wan (2009), "Regulating Risk or Risking Regulation? Construal Levels and Depletion Effects in the Processing of Health Messages," Journal of Consumer Research, 36 (3), 448-62.

$\rightarrow$ Albarracín, Dolores, and Amy L. Mitchell (2004), "The Role of
Defensive Confidence in Preference for Proattitudinal Information: How Believing That One Is Strong Can Sometimes Be a Defensive Weakness," Personality and Social Psychology Bulletin, 30 (12), 1565-84.

$\rightarrow$ Alexander David L., John Lynch, and Qing Wang (2008), “As Time Goes By: Do Cold Feet Follow Warm Intentions for Really New versus Incrementally New Products?" Journal of Marketing Research, 45 (3), 307-19.

$\rightarrow$ Amit, Elinor, Daniel Algom, and Yaacov Trope (2009), "DistanceDependent Processing of Pictures and Words," Journal of Experimental Psychology: General, 138 (3), 400-415. 
Briñol, Pablo, Richard E. Petty, Carmen Valle, Derek D. Rucker, and Alberto Becerra (2007), "The Effects of Message Recipients' Power before and after Persuasion: A Self-Validation Analysis," Journal of Personality and Social Psychology, 93 (6), 1040-53.

Cacioppo, John T., and Richard E. Petty (1981), "Social Psychological Procedures for Cognitive Response Assessment: The Thought Listing Technique," in Cognitive Assessment, ed. Merluzzi Thomas, Carol R. Glass, and Myles Genest, New York: Guilford, 309-42.

Chaiken, Shelly, Akiva Liberman, and Alice H. Eagly (1989), "Heuristic and Systematic Processing within and beyond the Persuasion Context," in Unintended Thought, ed. James S. Uleman and John A. Bargh, New York: Guilford, 212-52.

$\rightarrow$ Collins, Allan M., and Elizabeth F. Loftus (1975), "A SpreadingActivation Theory of Semantic Processing," Psychological Review, 82 (November), 407-28.

$\rightarrow$ Correll, Joshua, Steven J. Spencer, and Mark P. Zanna (2004), "An Affirmed Self and an Open Mind: Self-Affirmation and Sensitivity to Argument Strength," Journal of Experimental Social Psychology, 40 (3), 350-56.

$\rightarrow$ DeBono, Kenneth G., and Michelle Packer (1991), "The Effects of Advertising Appeal on Perception of Product Quality," Personality and Social Psychology Bulletin, 17 (2), 194-200.

$\rightarrow$ Edwards, John A. (2003), "The Interactive Effects of Processing Preference and Motivation on Information Processing: Causal Uncertainty and the MBTI in a Persuasion Context," Journal of Research in Personality, 37 (2), 89-99.

$\rightarrow$ Fujita, Kentaro, Yaacov Trope, Nira Liberman, and Maya LevinSagi (2006), "Construal Levels and Self-Control," Journal of Personality and Social Psychology, 90 (3), 351-67.

$\rightarrow$ Gal, David, and Derek D. Rucker (2011), "Answering the Unasked Question: Response Substitution in Consumer Survey," Journal of Marketing Research, 48 (1), 185-95.

$\rightarrow$ Gilbert, Daniel T., and Patrick S. Malone (1995), "The Correspondence Bias," Psychological Bulletin, 117 (1), 21-38.

$\rightarrow$ Griffin, Dale W., David Dunning, and Lee Ross (1990), "The Role of Construal Processes in Overconfident Predictions about Self and Others," Journal of Personality and Social Psychology, 59 (6), 1128-39.

$\rightarrow$ Hamann, Stephan B. (1990), "Level-of-Processing Effects in Conceptually Driven Implicit Tasks," Journal of Experimental Psychology: Learning, Memory, and Cognition, 16 (November), 970-77.

$\rightarrow$ Johnson, Michael D. (1984), "Consumer Choice Strategies for Comparing Noncomparable Alternatives," Journal of Consumer Research, 11 (December), 741-53.

Kruglanski, Arie W. (1989), Lay Epistemics and Human Knowledge: Cognitive and Motivational Bases, New York: Plenum.

$\rightarrow$ Labroo, Aparna A., and Vanessa M. Patrick (2009), "Providing a Moment of Respite: Why a Positive Mood Helps Seeing the Big Picture," Journal of Consumer Research, 35 (5), 800 809.

$\rightarrow$ Liberman, Nira, Michael D. Sagristano, and Yaacov Trope (2002), "The Effect of Temporal Distance on Level of Mental Construal," Journal of Experimental Social Psychology, 38, 523-34.

$\rightarrow$ Liberman, Nira, and Yaacov Trope (1998), "The Role of Feasibility and Desirability Considerations in Near and Distant Future Decisions: A Test of Temporal Construal Theory," Journal of Personality and Social Psychology, 75 (July), 5-18.

$\rightarrow$ Lyubomirsky, Sonja, Laura King, and Ed Diener (2005), "The
Benefits of Frequent Positive Affect: Does Happiness Lead to Success?" Psychological Bulletin, 131 (6), 803-55.

$\rightarrow$ Maheswaran, Durairaj, and Shelly Chaiken (1991), "Promoting Systematic Processing in Low-Motivation Settings: Effect of Incongruent Information on Processing and Judgment," Journal of Personality and Social Psychology, 61 (1), 13-25.

$\rightarrow$ Nussbaum, Shiri, Yaacov Trope, and Nira Liberman (2003), "Creeping Dispositionism: The Temporal Dynamics of Behavior Prediction," Journal of Personality and Social Psychology, 84 (3), 485-97.

$\rightarrow$ Park, C. Whan, and Daniel C. Smith (1989), "Product-Level Choice: A Top-Down or Bottom-Up Process?" Journal of Consumer Research, 16 (December), 289-99.

Petty, Richard E., Pablo Briñol, Zakary L. Tormala, and Daniel T. Wegener (2007), "The Role of Metacognition in Social Judgment," in Social Psychology: Handbook of Basic Principles, 2nd ed., ed. Arie W. Kruglanski and E. Tory Higgins, New York: Guilford, 254-84.

Petty, Richard E., and John T. Cacioppo (1986), Communication and Persuasion: Central and Peripheral Routes to Attitude Change, New York: Springer.

Petty, Richard E., and Duane T. Wegener (1998), “Attitude Change: Multiple Roles for Persuasion Variables," in The Handbook of Social Psychology, 4th ed., Vol. 1, ed. Daniel Gilbert, Susan T. Fiske, and Gardner Lindzey, New York: McGraw-Hill, 323-90.

$\rightarrow$ Pieters, Rik, and Michel Wedel (2004), "Attention Capture and Transfer in Advertising: Brand, Pictorial, and Text-Size Effects," Journal of Marketing, 68 (April), 36-50.

$\rightarrow$ Preacher, Kristopher J., Derek D. Rucker, and Andrew Hayes (2007), "Addressing Moderated Mediation Hypotheses: Theory, Methods, and Prescriptions," Multivariate Behavioral Research, 42 (1), 185-227.

$\rightarrow$ Rucker, Derek D., and Adam D. Galinsky (2008), "Desire to Acquire: Powerlessness and Compensatory Consumption," Journal of Consumer Research, 35 (2), 257-67.

$\rightarrow$ Rucker, Derek D., and Richard E. Petty (2006), "Increasing Effectiveness of Communications to Consumers: Recommendations Based on the Elaboration Likelihood and Attitude Certainty Perspectives," Journal of Public Policy and Marketing, 25 (1), 39-52.

Rucker, Derek D., Richard E. Petty, and Joseph R. Priester (2007), "Understanding Advertising Effectiveness from a Psychological Perspective: The Importance of Attitudes and Attitude Strength," in The Handbook of Advertising, ed. Gerard J. Tellis and Tim Ambler, Thousand Oaks, CA: Sage, 71-88.

Schwarz, Norbert (2002), "Situated Cognition and the Wisdom of Feelings: Cognitive Tuning," in The Wisdom in Feelings, ed. Lisa Feldman Barrett and Peter Salovey, New York: Guilford, 144-66.

___ (2004), "Metacognitive Experiences in Consumer Judgment and Decision Making," Journal of Consumer Research, 14 (4), 332-48.

$\rightarrow$ Sherman, Steven J. (1980), "On the Self-Erasing Nature of Errors of Prediction," Journal of Personality and Social Psychology, 39 (2), 211-21.

Shrout, Patrick E., and Niall Bolger (2002), "Mediation in Experimental and Nonexperimental Studies: New Procedures and Recommendations," Psychological Methods, 7 (4), 422-45.

$\rightarrow$ Smith, Pamela K., and Yaacov Trope (2006), "You Focus on the Forest When You're in Charge of the Trees: Power Priming and Abstract Information Processing," Journal of Personality and Social Psychology, 90 (4), 578-96. 
Sujan, Mita (1985), "Consumer Knowledge: Effects on Evaluation Strategies Mediating Consumer Judgments," Journal of Consumer Research, 12 (1), 31-46.

$\rightarrow$ Tiedens, Larissa Z., and Susan Linton (2001), "Judgment under Emotional Certainty and Uncertainty: The Effects of Specific Emotions on Information Processing," Journal of Personality and Social Psychology, 81 (6), 973-88.

$\rightarrow$ Tormala, Zakary L., Derek D. Rucker, and Charles R. Seger (2008), "When Increased Confidence Yields Increased Thought: A Confidence-Matching Hypothesis," Journal of Experimental Social Psychology, 44 (January), 141-47.

$\rightarrow$ Trope, Yaacov, and Nira Liberman (2003), "Temporal Construal," Psychological Review, 110 (July), 403-21.

$\rightarrow$ Trope, Yaacov, Nira Liberman, and Cheryl Wakslak (2007), "Construal Levels and Psychological Distance: Effects on Representation, Prediction, Evaluation, and Behavior," Journal of Consumer Psychology, 17 (2), 83-95.

$\rightarrow$ Vallacher, Robin R., and Daniel M. Wegner (1987), "What Do People Think They're Doing? Action Identification and Human Behavior," Psychological Review, 94 (1), 3-15.

$\rightarrow$ _ (1989), "Levels of Personal Agency: Individual Variation in Action Identification," Journal of Personality and Social Psychology, 57 (4), 660-71.
Vallacher, Robin R., Daniel M. Wegner, and Maria P. Somoza (1989), "That's Easy for You to Say: Action Identification and Speech Fluency," Journal of Personality and Social Psychology, 56 (2), 199-208.

$\rightarrow$ Wan, Echo Wen, Derek D. Rucker, Zakary L. Tormala, and Joshua J. Clarkson (2010), "The Effect of Regulatory Depletion on Attitude Certainty," Journal of Marketing Research, 47 (3), 531-41.

$\rightarrow$ Wänke, Michaela, Herbert Bless, and Barbara Biller (1996), "Subjective Experience versus Content of Information in the Construction of Attitude Judgments," Personality and Social Psychology Bulletin, 22 (11), 1105-13.

$\rightarrow$ Weary, Gifford, and Jill A. Jacobson (1997), "Causal Uncertainty Beliefs and Diagnostic Information Seeking," Journal of Personality and Social Psychology, 73 (4), 839-48.

$\rightarrow$ Wood, Stacy, and John G. Lynch (2002), "Prior Knowledge and Complacency in New Product Learning," Journal of Consumer Research, 29 (3), 416-26.

$\rightarrow$ Zhao, Min, and Jinhong Xie (2011), "Effects of Social and Temporal Distance on Consumers' Responses to Peer Recommendations," Journal of Marketing Research, 48 (June), 486-96. 